\title{
Modellierbarer Hautschutzring
}

\section{Zweifacher Schutz, einfache Anwendung}

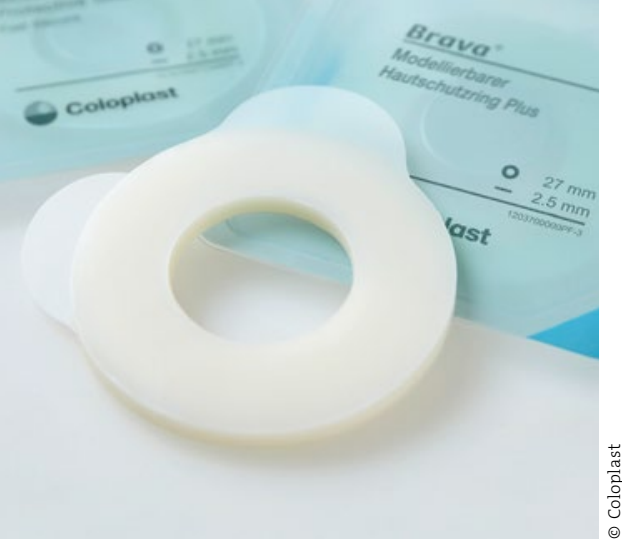

" Ganz gleich, ob kleine oder Stomata mit großem Durchmesser abzudichten sind, ob eher leichte oder starke Unebenheiten auszugleichen sind, um Le- ckagen zu vermeiden, oder ob irritierte Haut geschützt werden soll: Mit dem Brava $^{\circledR}$ Modellierbaren Hautschutzring Plus gibt es jetzt ein ganzes Ringsortiment, um so für jedes Stoma einen passenden Ring anbieten zu können. Dank spezieller Rezeptur bietet er die ideale Kombination aus Absorptionsvermögen und Beständigkeit. Durch die BodyFit ${ }^{\circledR}$ Technologie hat der neue Hautschutzring eine hohe Anpassungsfähigkeit, die für eine zuverlässige Abdichtung sorgt. Dank einer neuen Polymerformel ist er erosionsbeständig und löst sich auch nach lang anhaltendem Kontakt mit aggressiven Ausscheidungen nicht auf. Denn aufgrund der speziellen Rezeptur kann der Ring hohe Mengen an Feuchtigkeit absorbieren. Er hat eine sehr hautfreundliche Zusammensetzung, so dass er auch auf feuchter oder gereizter Haut eingesetzt werden kann.

Das Besondere: Der Brava ${ }^{\circledR}$ Modellierbarer Hautschutzring Plus lässt sich unkompliziert in die gewünschte Form und Größe modellieren. Er behält die Form auch während des Tragens selbst bei starker Bewegung. Der Ring ist extrem vielfältig in der Anwendung: Der Stomaträger kann ihn bei Bedarf zu einer Rolle formen oder durchschneiden. Er lässt sich stapeln, etwa um Konvexitäten zu verstärken, oder schön ausformen, um Vertiefungen auszukleiden. Und noch ein großes Plus: Der Hautschutzring löst sich zusammen mit der Stomaplatte ab, wobei sich eventuelle Rückstände einfach entfernen lassen.

Der Brava ${ }^{\circledR}$ Modellierbare Hautschutzring Plus ist in zwei Stärken ( 2,5 und 4,2 $\mathrm{mm}$ ) und drei verschiedenen Starterlochgrößen (18, 27 und $34 \mathrm{~mm})$ erhältlich. Dies ermöglicht eine einfache Anwendung und sichere Abdichtung auch bei größeren Stomadurchmessern, doppelläufigen Stomata und unterschiedlichsten Stomaumgebungen.

\section{www.bravaplus.coloplast.de}

\section{Vorlagen, Pull-ups \& Co}

\section{Neue Testsieger bei Inkontinenzprodukten}

» Egal, ob Krankenhaus, Pflegeheim oder Pflege zuhause - die Nutzung der richtigen, qualitativ hochwertigen Inkontinenzprodukte entscheidet über das Wohlbefinden des Patienten, aber auch den Aufwand für Pflegekräfte. Slips oder Vorlagen müssen verschiedenste Ansprüche verbinden. Sie sollen u.a. saugkräftig sein, ein Auslaufen verhindern und dadurch auch die Haut der Betroffenen schonen. Rund $200 \mathrm{~Pa}-$ tienten testeten im Auftrag der Stiftung Warentest in drei Kategorien Produkte der verschiedenen Hersteller.

In der Gruppe der hochaufnahmefähigen Inkontinenzslips, die alle in der Saugleistung nach gängigen Messmethoden auf ähnlichem Niveau liegen und damit vergleichbar sind, schloss MoliCare Premium Slip super plus L mit dem Qualitätsurteil „Gut“ (Note 2,1) als Testsieger ab. Die Probanden verwiesen hier auf das sichere Gefühl, das bei dem Tabuthema Inkontinenz maßgeblich ist, sowie den Tragekomfort. Neben der reinen Praktikabilität des Slips zeichnet sich MoliCare Premium auch durch klare und einfache Informationen auf der Packung aus.

Die Verständlichkeit ist auch ein Pluspunkt des Testsiegers MoliForm Premium extra soft Inkontinenz Einlagen, die mit der Gesamtnote 1,9 (Gut) bewertet wurden. Auch in der Kategorie der Vorlagen wurden nur vergleichbare, hochaufnahmefähige Produkte getestet. Gegenüber qualitativ schwächeren Vorlagen, verfügt MoliForm Premium über einen guten Auslaufschutz sowie Tragekomfort. Außerdem führt das Produkt nicht zu einem Austrocknen der Haut, was im schlimmsten Fall zu einem erheblichen Pflegeaufwand für Fachkräfte führen kann. „Stiftung Warentest macht deutlich, dass das Angebot der Markenhersteller gegenüber Produkten, die bei den Ausschreibungen der Kran- kenkassen oder niedrigen Pauschalen angeboten werden, qualitativ wesentlich besser ist. Hier bedarf es mittelfristig eines Umdenkens der Kassen“, fügt Raimund Koch, Referat Gesundheitspolitik bei der PAUL HARTMANN AG hinzu.

Einmalhosen - Pull-ups - eignen sich vor allem für demente Patienten, die andere Hilfsmittel oft ablehnen. Aber auch Menschen, die noch aktiv sind, nutzen diese Produkte, da sie sehr unauffällig sind. MoliCare Mobile überzeugte im Test durch die größte Aufnahmemenge $(630 \mathrm{ml})$ und erhielt das Testurteil „Gut“ (Note 2,0). „Der Test zeigt, dass sich Qualität auszahlt. Nicht nur der Patient kann mit der Krankheit besser umgehen, auch Pflegekräfte werden durch Qualitätsprodukte entlastet", berichtet Robin Bähr. 